\title{
Right Pulmonary Vein
}

National Cancer Institute

\section{Source}

National Cancer Institute. Right Pulmonary Vein. NCI Thesaurus. Code C48947.

A vein that returns oxygenated blood from the right lung to the left atrium of the heart. 